\title{
Estrategia integral de formación para fortalecer las competencias de la educación en la ingeniería.
}

DOI: 10.13140/RG.2.2.19300.40324

Presentado por: Jaime Augusto Pinzón Mendieta.

\section{Resumen:}

En la actualidad, las tecnologías de la información y las comunicaciones (TIC) han generado alto impacto en el desarrollo de la humanidad cambiando la relación de cada individuo con su entorno. En el sector educativo, el estudiante y el docente han tenido cambios significativos en sus procesos de enseñanza-aprendizaje y su relación con la sociedad del conocimiento. El objetivo del presente trabajo, es demostrar que la apropiación de conocimiento de los estudiantes, el trabajo colaborativo con los docentes y el apoyo de la institución de educación superior; generan soluciones asertivas para las necesidades de un contexto especifico. Se utiliza una metodología formativa aplicando buenas prácticas para la gestión de proyectos integrando las competencias del plan de estudios del programa de ingeniería de sistemas. Conclusiones: integrar y fortalecer las competencias del estudiante en las dimensiones del ser, conocer, hacer y convivir, le permitirá ser más competitivo en el actual mercado con una actitud responsable, emprendedora e investigativa.

\section{Comprehensive training strategy to strengthen the skills of engineering education}

\begin{abstract}
:
Currently, information and communication technologies (TIC) have generated a high impact on the development of humanity by changing the relationship of each individual with their environment. In the education sector, the student and the teacher have had significant changes in their teaching-learning processes and their relationship with the knowledge society. The objective of this work is to demonstrate that the appropriation of knowledge by students, collaborative work with teachers and the support of the higher education institution; they generate assertive solutions for the needs of a specific context. A training methodology is used, applying good practices for project management, integrating the competencies of the syllabus of the systems engineering program. Conclusions: integrating and strengthening the student's skills in the dimensions of being, knowing, doing and living together, will allow him to be more competitive in the current market with a responsible, entrepreneurial and investigative attitude.
\end{abstract}

Palabras clave: aprendizaje, gestión, formación, TIC, estrategia. 
Key words: learning, management, training, TIC, strategy.

Tema: Proyecto integrador o proyecto articulador

\section{Contexto Internacional:}

- ¿Quiénes son los países líderes en la investigación y producción científica del mundo en el tema? Top 10 Fuente: Scimago

Respuesta: Por ser un tema de innovación curricular y cruzando otras bases de datos, los países que han trabajado ese tema son México, Chile y Venezuela.

- ¿Qué puesto ocupa Colombia en la producción científica mundial y regional sobre el tema? Fuente: Scimago Respuesta: Ocupa el segundo puesto en América del sur entre dos países.

- ¿Cuáles son las publicaciones más importantes a nivel mundial y regional en el tema? Top 10 Fuente: Scimago

Respuesta: Publicaciones relevantes en el campo mundial, no existen. En el contexto regional está la Universidad de los Andes, Universidad de Antioquia.

- En ¿Qué año se han publicado más artículos de acuerdo con la ecuación de búsqueda sobre el tema? Fuente: Scopus

Respuesta: En el año 2017 se publicaron 14 artículos, seguido de 13 artículos en el 2018

- ¿Quiénes son los autores con más producción de acuerdo con la ecuación de búsqueda sobre el tema? Top 10 Fuente: Scopus

Respuesta: Omar Abreu, Sergio Godoy, Myrna Gálvez y Claudia Labarca.

- ¿Cuáles son las instituciones que más han apoyado las publicaciones de acuerdo con la ecuación de búsqueda sobre el tema? Top 10 Fuente: Scopus

Respuesta: Pontificia Universidad Católica de Chile y la Escuela de Comunicaciones de la PUC ambas en Chile y la universidad Técnica del Norte, Ibarra, Ecuador.

- ¿Cuál es la tipología documental que más se ha publicado de acuerdo con la ecuación de búsqueda? Fuente: Scopus

Respuesta: 65 artículos de 144 búsquedas entre otros productos de nuevo conocimiento. 


\section{Contexto regional:}

- ¿Qué países lideran la producción académica en Latinoamérica de acuerdo con la ecuación de búsqueda? Fuente: LaReferencia Respuesta: La lidera Perú con 24 publicaciones, le sigue Colombia con 14 publicaciones y finalmente Costa rica, Argentina y Ecuador con 11, 10 y 8 respectivamente.

- ¿Quiénes son los 5 autores que más publican en Latinoamérica de acuerdo con la ecuación de búsqueda? Fuente: LaReferencia Respuesta: Gatica-Chandia, Miguel, Guzmán-Ovares, Marcela y Justo Estebaranz, Jesús con 2 publicaciones cada uno.

- ¿Cuáles son las universidades que más publican en Latinoamérica de acuerdo con la ecuación de búsqueda? Fuente: LaReferencia Respuesta: Universidad Nacional de ingeniería, Universidad de los Andes y Autoridad Nacional del Agua

- ¿Cuál es la tipología documental de mayor publicación en Latinoamérica de acuerdo con la ecuación de búsqueda? Fuente: LaReferencia Respuesta: Tesis de maestría con 43 y artículos con 26 publicaciones.

\section{Estudio cienciométrico:}

\begin{tabular}{|l|l|l|c|c|c|}
\hline \multicolumn{1}{|c|}{ Indicador } & \multicolumn{1}{c|}{ Descripción } & \multicolumn{1}{c|}{ Fuente } & $\mathbf{2 0 1 8}$ & $\mathbf{2 0 1 9}$ & $\mathbf{2 0 2 0}$ \\
\hline Indicador de producción & Total de artículos & Scopus & 13 & 11 & 66 \\
\hline Indicador de producción & Total de libros & Scopus & 1 & 0 & 0 \\
\hline Indicador de producción & $\begin{array}{l}\text { Total de capítulos de } \\
\text { libros }\end{array}$ & Scopus & 0 & 0 & 0 \\
\hline Indicador de producción & $\begin{array}{l}\text { Total de Conference } \\
\text { Paper }\end{array}$ & Scopus & 0 & 0 & 0 \\
\hline Indicador de producción & $\begin{array}{l}\text { Principal entidad } \\
\text { financiadoras }\end{array}$ & Scopus & 0 & 0 & 0 \\
\hline Indicador de producción & $\begin{array}{l}\text { Principal país de } \\
\text { publicación (sin incluir } \\
\text { el país de origen) }\end{array}$ & Scopus & España & 0 & 0 \\
\hline Indicador de producción & $\begin{array}{l}\text { Autor con mayor } \\
\text { publicaciones }\end{array}$ & Scopus & 2 & 1 & 0 \\
\hline Indicador de producción & Total de artículos & WOS & 0 & 0 & 2 \\
\hline
\end{tabular}


Métricas de Investigación: Cienciometría, Bibliometría y Datos Abiertos

\begin{tabular}{|c|c|c|c|c|c|}
\hline Indicador de producción & Total de libros & WOS & 0 & 0 & 0 \\
\hline Indicador de producción & $\begin{array}{l}\text { Total de capítulos de } \\
\text { libros }\end{array}$ & WOS & 0 & 0 & 0 \\
\hline Indicador de producción & \begin{tabular}{|l|} 
Total de Conference \\
Paper
\end{tabular} & WOS & 0 & 0 & 0 \\
\hline Indicador de producción & \begin{tabular}{|l|} 
Principal entidad \\
financiadoras
\end{tabular} & WOS & 0 & 0 & 0 \\
\hline Indicador de producción & \begin{tabular}{|l|} 
Principal país de \\
publicación (sin incluir \\
el país de origen)
\end{tabular} & WOS & 0 & 0 & 0 \\
\hline Indicador de producción & \begin{tabular}{|l} 
Autor con mayor \\
publicaciones
\end{tabular} & WOS & 0 & 0 & 0 \\
\hline Indicador de producción & Total de artículos & LaReferencia & 10 & 16 & 0 \\
\hline Indicador de producción & Total de libros & LaReferencia & 0 & 0 & 0 \\
\hline Indicador de producción & Total Tesis Doctorado & LaReferencia & 0 & 1 & 0 \\
\hline Indicador de producción & Total de Maestría & LaReferencia & 21 & 22 & 0 \\
\hline Indicador de producción & $\begin{array}{l}\text { Principal país de } \\
\text { publicación (sin incluir } \\
\text { el país de origen) }\end{array}$ & LaReferencia & Perú & Perú & 0 \\
\hline Indicador de producción & $\begin{array}{l}\text { Autor con mayor } \\
\text { publicaciones }\end{array}$ & LaReferencia & 2 & 2 & 0 \\
\hline Indicador de producción & Total de artículos & Scielo & 0 & 0 & 0 \\
\hline Indicador de producción & $\begin{array}{l}\text { Principal entidad } \\
\text { financiadoras }\end{array}$ & Scielo & 0 & 0 & 0 \\
\hline Indicador de producción & $\begin{array}{l}\text { Principal país de } \\
\text { publicación (sin incluir } \\
\text { el país de origen) }\end{array}$ & Scielo & Chile & México & 0 \\
\hline Indicador de producción & $\begin{array}{l}\text { Autor con mayor } \\
\text { publicaciones }\end{array}$ & Scielo & $\begin{array}{l}\text { Patricio } \\
\text { Felmer }\end{array}$ & \begin{tabular}{|c|} 
Josefa \\
Perdomo \\
Díaz
\end{tabular} & 0 \\
\hline Indicador de producción & $\begin{array}{ll}\text { Total } & \text { de } \\
\text { publicaciones } & \end{array}$ & $\begin{array}{l}\text { Google } \\
\text { Scholar }\end{array}$ & \multicolumn{3}{|c|}{$\begin{array}{c}\text { Con respecto a tres palabras clase } \\
\text { según la fórmula de búsqueda, arroja } \\
18.000 \text { resultados. }\end{array}$} \\
\hline
\end{tabular}

El tema del proyecto articulador como estratégica formativa no es muy común en el contexto mundial ya en el sistema de información científica suministrado por Clarivate Analytics no hay registro en tema y título.

Sin embargo, en la base de datos bibliográfica de resúmenes y citas de artículos de revistas científicas Scopus se encontró 62 artículos en los últimos tres años con mayor producción en España con 8 articulos promedio por año como se logra apreciar en la Tabla 1. 
Tabla 1. Resultados de la búsqueda en Scopus

\begin{tabular}{|l|c|c|c|}
\hline & 2018 & 2019 & 2020 \\
\hline Articulos & 37 & 23 & 2 \\
\hline Libros & 1 & 0 & 0 \\
\hline Capitulos & 0 & 0 & 0 \\
\hline Conference Paper & 0 & 0 & 0 \\
\hline Entidad financiadora & 0 & 0 & 0 \\
\hline
\end{tabular}

Como estrategia formativa curricular, no existen entidades financiadoras ya que son estrategias impulsadas y respaldadas por las mismas instituciones educativas.

Con respecto a la visibilidad de la producción científica de las instituciones de educación superior e investigación de América Latina, LaReferencia en la Tabla 2, y aplicando la misma ecuación de búsqueda en Scopus, se encuentra mayor investigación realizada en los programas de maestría orientados a la integración de competencias específicas para desarrollar soluciones a las necesidades del contexto social con 41 publicaciones en los últimos 2 años y artículos en revistas indexadas con un 35\% menos que en Scopus.

Tabla 2. Resultados de búsqueda en LaReferencia

\begin{tabular}{|c|c|c|c|}
\hline Descripción & 2018 & 2019 & 2020 \\
\hline Articulos & 10 & 16 & 0 \\
\hline Libros & 0 & 0 & 0 \\
\hline $\begin{array}{l}\text { Tesis } \\
\text { Doctorales }\end{array}$ & 0 & 1 & 0 \\
\hline $\begin{array}{ll}\text { Tesis } & \text { de } \\
\text { Maestria } & \\
\end{array}$ & 21 & 22 & 0 \\
\hline
\end{tabular}

\begin{tabular}{|l|lr|l|c|c|c|}
\hline Indicador & Descripción & Fuente & 2018 & 2019 & 2020 \\
\hline Índice de actividad & $\begin{array}{l}\text { tema, área de } \\
\text { conocimiento } \\
\text { disciplina }\end{array}$ & $\begin{array}{l}\text { y/o } \\
\text { tema, área de }\end{array}$ & Scopus & 0 & 0 & 0 \\
\hline Índice de actividad & $\begin{array}{l}\text { conocimiento } \\
\text { disciplina }\end{array}$ & wos & 0 & 0 & 0 \\
\hline
\end{tabular}


Métricas de Investigación: Cienciometría, Bibliometría y Datos Abiertos

\begin{tabular}{|c|c|c|c|c|c|}
\hline Tasa de crecimiento & $\left|\begin{array}{lr}\text { tema, área } & \text { de } \\
\text { conocimiento } & \text { y/o } \\
\text { disciplina } & \end{array}\right|$ & Scopus & 0 & 0 & 0 \\
\hline Tasa de crecimiento & $\begin{array}{|lr|}\text { tema, área } & \text { de } \\
\text { conocimiento } & \text { y/o } \\
\text { disciplina } & \end{array}$ & WOS & 0 & 0 & 0 \\
\hline Índice de impacto & Revistas en Q1 & Scimago & 0 & 0 & 0 \\
\hline Índice de impacto & Revistas en Q2 & Scimago & 0 & 0 & 0 \\
\hline Índice de impacto & Revistas en Q3 & Scimago & 0 & 0 & 0 \\
\hline Índice de impacto & Revistas en Q4 & Scimago & 1 & 0 & 0 \\
\hline Índice de impacto & Revistas en Q1 & JCR & 0 & 0 & 0 \\
\hline Índice de impacto & Revistas en Q2 & JCR & 0 & 0 & 0 \\
\hline Índice de impacto & Revistas en Q3 & JCR & 0 & 0 & 0 \\
\hline Índice de impacto & Revistas en Q4 & JCR & 1 & 0 & 0 \\
\hline
\end{tabular}

\section{Conclusiones:}

Los retos administrativos y académicos que se deben superar en las estrategias formativas integradoras que mejoren las propuestas de productos resultados de investigación en la formación de recurso humano; consiste en habilitar espacios de trabajo de pares que permitan unificar criterios de los estudiantes con el compromiso de responsabilidad social que tienen los docentes en sus reglamentos o estatutos, ya que son dinamizadores principales de estas iniciativas.

Los espacios académico presenciales y virtuales con pensamiento crítico y reflexivo evidencia la necesidad de generar tiempos adicionales de asesorías y talleres especializados en gestión de proyectos e investigación.

Es necesario incentivar la generación de redes de negocio que articulen las TIC y el conocimiento con apoyos de financiación del sector público o privado. Además, es importante motivar la comunidad académica para desarrollar, articular y dinamizar las estrategias de ciencia, tecnología en innovación promoviendo cultura en investigación en un marco de ética, bioética e integridad académica.

Estos escenarios y otros factores de carácter institucional reflejan que aún existen debilidades que se deben analizar para desarrollar estrategias formativas con 
mejores competencias específicas para desarrollar soluciones articuladas con el sector empresarial y social.

\section{Referencias.}

- Congreso de la república de Colombia. (1998) .Ley general de educación. Disponible en: http://www.mineducacion.gov.co/1621/articles85906_archivo_pdf.pdf

- Congreso de la república de Colombia. (2206). Ley 1014, de fomento a la cultura del emprendimiento. Disponible en: https://www.mincomercio.gov.co/publicaciones.php?id=14257

- Moreno Méndez, Jaime, \& Rodríguez Quintero, Gloria, \& Mera Rodríguez, Edith, \& Beltrán Vargas, Luz (2007). Estrategias Didácticas Desarrolladas Por Los Docentes Para Orientar El Trabajo Integrador En La Facultad De Psicología. Avances de la disciplina, 1 (1), 133-162. [Fecha de consulta 2 de junio de 2020]. ISSN: 1900-2386. Disponible en: https://www.redalyc.org/articulo.oa?id=2972/297224869005

- Jaimes, F. (2010). El proyecto integrador: una estrategia pedagógica para la formación integral de los estudiantes. Desarrollo \& Gestión, (5). Recuperado a partir de https://revistas.uniminuto.edu/index.php/DYG/article/view/466

- Coordinación de formación Facultad de Ciencias Admiistrativas Universidad de Guayaquil. (2016). Instructivo para el desarrollo de proyectos integradores a través de la catedra integradora. Guayaquil: UG.

- Project Management Institute PMI. (2015). Guía de los fundamentos para la dirección de proyectos (Guía del PMBOK). Sexta edición. Newtown Square, Pennsylvania, EUA : PMI®. pp. 43, 103-312, 467

- Proyecto integrador como estrategia formativa para el fortalecimiento de competencias específicas y transversales en la facultad de ingeniería 9 weeftm 2013 cartagena.

- Revelo, C. \& Rivas, V. (2008). El proyecto integrador como investigación en el aula. Fundación Academia de Dibujo Profesional. Programa de Dibujo Arquitectónico y Decoración. I congreso Latinoamericano de enseñanza en diseño. Universidad de Palermo.

- Aguilera Cruz, O. y Ruiz de la Peña, J. (2006): Importancia de la Ingeniería de Software en la producción de software. Revista electrónica "Ciencias Holguín" ISSN 1027-2127. htttp://www. ciencias.holguín.cu, número 4.

- Hewitt Ramirez, Nohelia (2007). El Proyecto Integrador: Una Estrategia Pedagógica Para Lograr La Integración Y La Socialización Del Conocimiento. Psicologia. Avances de la disciplina, 1 (1), 235-240. [Fecha de consulta 2 de junio de 2020]. ISSN: 1900-2386. Disponible en: https://www.redalyc.org/articulo.oa?id=2972/297224869006 